\title{
Design and Test of Discrete Time Adaptive Backup Flight Control Laws
}

\author{
Andreas Myleus* Daniel Simon** Peter Rosander*** \\ * Saab AB, SE-581 83 Linköping, Sweden (e-mail: \\ andreas.myleus@saabgroup.com) \\ ** Saab AB, SE-581 83 Linköping, Sweden (e-mail: \\ daniel.simon@saabgroup.com) \\ *** Saab AB, SE-581 83 Linköping, Sweden (e-mail: \\ peter.rosander@saabgroup.com)
}

\begin{abstract}
This paper is the result of a Master Thesis project performed at Saab AB. The main goal of the project was to design and implement a discrete time $\mathcal{L}_{1}$ adaptive back-up controller in pitch to increase performance for an agile fighter aircraft. A lateral controller was also developed as a secondary goal.

A discrete time version of the Modified Piecewise Constant $\mathcal{L}_{1}$ adaptive control formulation was implemented. Results have shown that augmenting a state-feedback controller with an $\mathcal{L}_{1}$ adaptive controller increases robustness in the whole flying envelope, with satisfactory flying qualities. A switching scheme between two $\mathcal{L}_{1}$ controllers based on the extension of the landing gear was used to improve the controller performance throughout the whole envelope. The implemented controllers were flown in a simulator with a nonlinear generic fighter aircraft model with promising results.
\end{abstract}

Keywords: Adaptive control, Flight control, Aircraft control, Back-up controllers

\section{INTRODUCTION}

The dynamics of an aircraft vary considerably in different parts of the flight envelope. To deal with this, gain scheduling of multiple linear controllers, have traditionally been used. Typically this requires that the current speed and altitude of the aircraft are measurable. If the systems which measure speed and altitude fail, a backup system with a controller that can adjust to that situation is required. In recent years, various methods for adaptive control have grown increasingly popular since they can automatically adapt to the varying dynamics of the aircraft.

The task was to investigate the $\mathcal{L}_{1}$ adaptive method with respect to aircraft control and loss of airspeed and altitude data. In other words, it will have to be able to compensate for the lack of air data information (such as dynamic pressure), but also, e.g., model errors and nonlinearities. Another requirement is that it would also need to compensate for changing control surface authority across the envelope.

For practical reasons it would also need to be implementable digitally with discrete-time models, control law and adaptation law with a sampling frequency of $60 \mathrm{~Hz}$.

\subsection{The flight dynamic model}

The aircraft model used in this project is called VEGAS and consists of a nonlinear aerodata model, rigid body dynamics, servo dynamics, engine model, atmospheric model, and so on. The aerodynamic derivatives used in VEGAS are part of a generic fighter jet model known as
ADMIRE (AeroDATA Model In Research Environment). This model was jointly developed by SAAB, the Royal Institute of Technology and the Swedish Defence Research Agency [ADM]. The ADMIRE model is open for anyone to use and similar to the Gripen fighter aircraft, as it includes the same types of control surfaces and has similar characteristics, such as unstable pitch dynamics in the subsonic region.

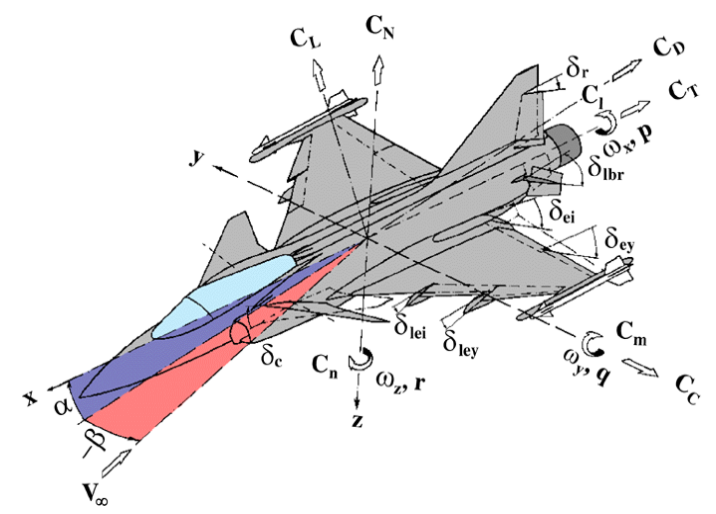

Fig. 1. Body- and Wind-axes coordinate system of a Gripen fighter aircraft with control surfaces, rotational rates, angles and non-dimensional aerodynamic forces and moments. [Courtesy of SAAB].

The full VEGAS model has 9 states, 13 inputs and 28 outputs, which is excessive for the purpose of this project. To capture the relevant dynamics for the problem we define the states and control as 


$$
\begin{aligned}
& x=[\beta, \alpha, p, q, r]^{T} \\
& u=\left[\delta_{e}, \delta_{a}, \delta_{r}\right]^{T}
\end{aligned}
$$

where $\beta$ and $\alpha$ are the angles between the aircraft $\mathrm{x}$-axis and the speed vector; $p, q, r$ are the body angular rates and $\delta_{*}$ are the elevator, aileron and rudder control surface deflections, see Figure 1.

For simulation we have used the full nonlinear dynamical equations but the nonlinear model, with the relevant states, needs to be linearised in order to be used in the $\mathcal{L}_{1}$ adaptive control framework.

The linearisation from VEGAS, with a trimmed flight condition in Mach $=0.6$ and Altitude $=5000 \mathrm{~m}$, resulted in the following pitch dynamics

$$
\left[\begin{array}{c}
\dot{\alpha} \\
\dot{q}
\end{array}\right]=\left[\begin{array}{cc}
-1.16 & 0.926 \\
4.32 & -1.26
\end{array}\right]\left[\begin{array}{c}
\alpha \\
q
\end{array}\right]+\left[\begin{array}{c}
0.367 \\
28.4
\end{array}\right] \delta_{e}
$$

The corresponding equations for the lateral dynamics are

$$
\begin{aligned}
{\left[\begin{array}{c}
\dot{\beta} \\
\dot{p} \\
\dot{r}
\end{array}\right]=} & {\left[\begin{array}{ccc}
-0.247 & 0.048 & -0.944 \\
-25.1 & -2.23 & 0.484 \\
4.48 & -0.127 & -0.456
\end{array}\right]\left[\begin{array}{l}
\beta \\
p \\
r
\end{array}\right] } \\
& +\left[\begin{array}{cc}
-0.051 & 0.068 \\
56.3 & 5.58 \\
5.31 & -5.67
\end{array}\right]\left[\begin{array}{l}
\delta_{a} \\
\delta_{r}
\end{array}\right]
\end{aligned}
$$

\subsection{Adaptive flight control}

As stated above, an essential part of control design is to have knowledge of the system to be controlled. However, it is impossible to develop models which includes all the dynamics of a system.

The idea behind adaptive control is to design the controller for a single flight condition, and adapt to the changes of the system on-line. The changes are formulated as nonlinear parameter variations to a linear system. In this application, control surface deflections mainly produce aerodynamic moments which results in angular velocity, thus body rates $p, q$ and $r$ correspond to the matched uncertainties. An error in mass, mismodelling of aerodynamic coefficients or lack of air data information (such as dynamic pressure) however produce unmatched model errors.

One usually distinguish between two types of architectures of adaptive control, direct and indirect methods. In the direct methods the control parameters are estimated directly, while in the indirect methods the system parameters are estimated on-line, and the control parameters are obtained from a design procedure.

MRAC (Model Reference Adaptive Control) is an example of an adaptive control strategy, which can be implemented in either direct or indirect form. $\mathcal{L}_{1}$ was born from a reformulation of MRAC [1] with the addition of a lowpass filter in the feedback loop. The benefit of this was that one could design the controller not to let high frequency adaptation content to affect the control signal.

\section{2. $\mathcal{L}_{1}$ ADAPTIVE CONTROL}

Even though current control schemes obviously work they have their limitations. These limitations include the lack of robustness to changing system dynamics or unforeseen events such as actuator failure. An adaptive scheme could, e.g., relieve a baseline controller, where in the nominal case it would behave as usual, but in an unforeseen event the adaptive controller would extend the robustness. However, up until recently adaptive schemes have not been able to prove robustness a priori and have relied on MonteCarlo simulations to determine the best adaptation rate for various flight conditions [6]. Fast adaptation often led to high frequencies in control signals and increased sensitivity to time delays.

The philosophy of the $\mathcal{L}_{1}$ adaptive controller is to decouple the adaptation and the control loops by using a predictorbased fast adaptation scheme, still only compensating for the uncertainties within the bandwidth of the control channel. The estimation rate is only limited by the available CPU power, while the robustness is limited by the available control bandwidth.

There exist different types of $\mathcal{L}_{1}$ fast adaptation schemes; the gradient descent adaptive law, which we will not consider in this paper, the method described in [5], which achieves fast adaptation by increasing the adaptive gain, and also the piecewise constant adaptive law [5, 7], which achieves the same goal by increasing the sampling frequency. It is the later that we will explore in this paper.

By increasing the adaptation rate, the input and output of the system can be rendered arbitrarily close to the corresponding signals of the reference system that defines the desired closed-loop response, in both transient and steady state. The architecture does not require persistence of excitation, gain scheduling, control reconfiguration or a high-gain feedback controller [6]. In general you can say that the $\mathcal{L}_{1}$ adaptive controller, based on a linear identified system on the form

$$
\dot{x}(t)=A_{i d} x(t)+B_{i d} u(t)
$$

is designed to control a general unknown nonlinear system on the form,

$$
\begin{aligned}
& \dot{x}(t)=f_{x}(t, x)+B_{\text {true }}(t, x) u(t) \\
& y(t)=C_{m} x(t)
\end{aligned}
$$

The error between the systems above is identified on-line and corrected for by the controller.

As mentioned earlier, the architecture that have been chosen for the controller design is the modified piecewise constant $\mathcal{L}_{1}$ structure presented in [7], where the main difference from the controller in [13] is an additional term to the adaptive law. The controller in [13] has successfully been used in NASAs GTM (AirSTAR) [14] and the Boeing $\mathrm{X}-48 \mathrm{~B}[12]$.

\subsection{Piecewise constant $\mathcal{L}_{1}$ adaptive control architecture.}

The general structure of the $\mathcal{L}_{1}$ controller is shown in Figure 2. We will in this section describe the different parts of this structure.

Consider the true system formulation in (2), 


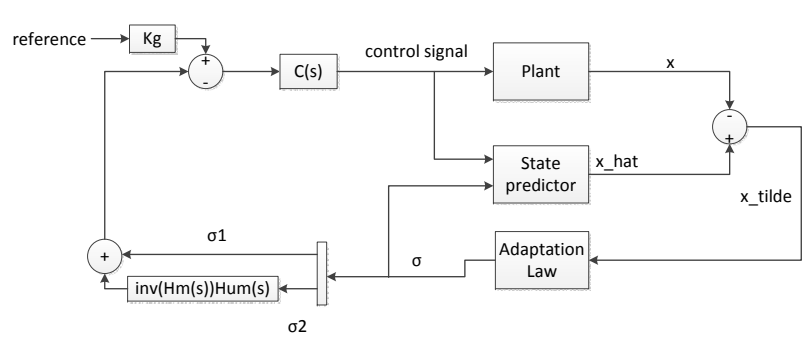

Fig. 2. Schematics of the $\mathcal{L}_{1}$ adaptive controller.

$$
\begin{aligned}
& \dot{x}(t)=f_{x}(t, x)+B_{\text {true }}(t, x) u(t) \\
& y(t)=C_{m} x(t)
\end{aligned}
$$

where $B_{\text {true }} \in \mathbb{R}^{n \times m}$ is the true unknown time-varying input matrix.

Also define,

$$
\begin{aligned}
f_{\Delta}(t, x) & =f_{x}(t, x)-A_{i d} x(t) \\
B_{\Delta}(t, x) & =B_{\text {true }}(t, x)-B_{i d} \omega
\end{aligned}
$$

where $A_{i d}$ and $B_{i d}$ are the identified linear system matrices, $f_{x}: \mathbb{R} \times \mathbb{R}^{n} \rightarrow \mathbb{R}^{n}$ and $f_{\Delta}: \mathbb{R} \times \mathbb{R}^{n} \rightarrow \mathbb{R}^{n}$ are unknown nonlinear functions, $B_{\Delta} \in \mathbb{R}^{n \times m}$ and $\omega \in \mathbb{R}^{m \times m}$ are unknown matrices. Substituting (3) and (4) to (2) gives the following identified system with model errors.

$$
\dot{x}(t)=A_{i d} x(t)+B_{i d} \omega u(t)+f_{\Delta}(t, x)+B_{\Delta}(t, x) u(t)
$$

Introducing a control signal with a nominal linear statefeedback part and an adaptive part and by considering assumptions and lemmas in [5] we can write

$$
u(t)=-\omega K_{x y} x(t)+\omega u_{a d}(t)+\sigma_{u}(t)
$$

inserting this into (5) and rewriting we obtain

$$
\begin{aligned}
\dot{x}(t) & =A_{i d} x(t)+B_{i d}\left(-\omega K_{x y} x(t)+\omega u_{a d}(t)+\sigma_{u}(t)\right) \\
& +f_{\Delta}(t, x)+B_{\Delta}(t, x)\left(-\omega K_{x y} x(t)+\omega u_{a d}(t)+\sigma_{u}(t)\right) \\
& =(\underbrace{A_{i d}-B_{i d} K_{x y}}_{A_{m}}) x(t)+B_{i d} K_{x y} x(t) \\
& +B_{i d}\left(-K_{x y} \omega x(t)+\omega u_{a d}(t)+\sigma_{u}(t)\right)+f_{\Delta}(t, x) \\
& +B_{\Delta}(t, x)\left(-\omega K_{x y} x(t)+\omega u_{a d}(t)+\sigma_{u}(t)\right) \\
& =A_{m} x(t)+B_{i d} \omega u_{a d}(t) \\
& +B_{i d}\left(K_{x y}(\mathbb{I}-\omega) x(t)+\sigma_{u}(t)\right)+f_{\Delta}(t, x) \\
& +B_{\Delta}(t, x)\left(-\omega K_{x y} x(t)+\omega u_{a d}(t)+\sigma_{u}(t)\right)
\end{aligned}
$$

where in the second equality we have added and subtracted $B_{i d} K_{x y} x(t)$. By defining

$$
\begin{aligned}
f(t, x) & \triangleq B_{i d}\left(K_{x y}(\mathbb{I}-\omega) x(t)+\sigma_{u}(t)\right)+f_{\Delta}(t, x) \\
& +B_{\Delta}(t, x)\left(-\omega K_{x y} x(t)+\omega u_{a d}(t)+\sigma_{u}(t)\right)
\end{aligned}
$$

and $B_{m} \triangleq B_{i d}$, we can write this as

$$
\dot{x}(t)=A_{m} x(t)+B_{m} \omega u_{a d}(t)+f(t, x)
$$

Now split $f(t, x)$ into two unknown nonlinear functions, $f_{1}: \mathbb{R}^{n} \times \mathbb{R} \rightarrow \mathbb{R}^{m}$ and $f_{2}: \mathbb{R}^{n} \times \mathbb{R} \rightarrow \mathbb{R}^{n-m}$ that satisfy

$$
\begin{aligned}
{\left[\begin{array}{l}
f_{1}(t, x(t)) \\
f_{2}(t, x(t))
\end{array}\right] } & =B^{-1} f(t, x(t)) \\
B & \triangleq\left[B_{m}, B_{u m}\right]
\end{aligned}
$$

The system (7) can then be written as

$$
\begin{aligned}
\dot{x}(t) & =A_{m} x(t)+B_{m}\left(\omega u_{a d}(t)+f_{1}(t, x(t))\right. \\
& +B_{u m} f_{2}(t, x) \\
y(t) & =C_{m} x(t)
\end{aligned}
$$

where $A_{m} \in \mathbb{R}^{n \times n}$ is a known Hurwitz matrix specifying the desired closed-loop dynamics, $B_{m} \in \mathbb{R}^{n \times m}$ is a full-rank known constant matrix, $B_{u m} \in \mathbb{R}^{n \times(n-m)}$ is a constant matrix such that $B_{m}^{T} B_{u m}=0$, and the rank of $\left[B_{m}, B_{u m}\right]=n, C_{m} \in \mathbb{R}^{m \times n}$ is a known full-rank constant matrix, $\left(A_{m}, B_{m}\right)$ is controllable and $\left(A_{m}, C_{m}\right)$ is observable. $\omega \in \mathbb{R}^{m \times m}$ is the uncertain system input gain matrix. In (10) $f_{1}$ represents the matched component of the unknown nonlinearities and $f_{2}$ represents the unmatched uncertainties.

We are now ready to formulate the different parts of the $\mathcal{L}_{1}$ adaptive controller.

\section{State-predictor}

By parametrizing the nonlinear functions $f_{1}(t, x(t))$ and $f_{2}(t, x(t))$ in (10) we obtain the following state-predictor

$$
\begin{aligned}
& \dot{\hat{x}}(t)=A_{m} \hat{x}(t)+B_{m}\left(\omega_{0} u_{a d}(t)+\hat{\sigma}_{1}(t)\right)+B_{u m} \hat{\sigma}_{2}(t)(11 \mathrm{a}) \\
& y(t)=C_{m} \hat{x}(t)
\end{aligned}
$$

where $\omega_{0}$ is the nominal system input gain, $\hat{\sigma}_{1}(t) \in \mathbb{R}^{m}$ and $\hat{\sigma}_{2}(t) \in \mathbb{R}^{(n-m)}$ are the adaptive matched and unmatched estimates. For each element in the input $u$ there is a matched $\hat{\sigma}$ and unmatched signals are added so that the total number of $\hat{\sigma}$ are equal to the number of states.

\section{Adaptation laws}

$$
\begin{aligned}
{\left[\begin{array}{c}
\hat{\sigma}_{1}(k) \\
\hat{\sigma}_{2}(k)
\end{array}\right] } & =\Phi\left(T_{s}\right)^{-1} h(k)-\Phi\left(T_{s}\right)^{-1} e^{A_{m} T_{s}} \tilde{x}(k) \\
\Phi\left(T_{s}\right) & =A_{m}^{-1}\left(e^{A_{m} T_{s}}-\mathbb{I}_{n}\right) B \\
h(k) & =-\tilde{x}(k)+h(k-1) \\
\tilde{x}(k) & \triangleq \hat{x}(k)-x(k)
\end{aligned}
$$

The time argument $k$ effectuates zero order sample and hold at sampling time intervals $T_{s}$, hence the name "piecewise constant". The adaptive laws is derived in [7] by integrating the error dynamics between the state predictor (11) and the true system (10).

\section{Control law}

To compensate for the uncertainties and track the reference signal $r(t)$ with zero steady-state error the control law in the frequency domain is given by

$$
\begin{aligned}
u(s)= & C(s)\left(K_{g} r(s)-\hat{\sigma}_{1}(s)-H_{m}^{-1}(s) H_{u m}(s) \hat{\sigma}_{2}(s)\right) \\
& -K_{x y} x(s)
\end{aligned}
$$

and the lowpass filter $C(s)$ is defined as

$$
C(s)=\omega K D(s)\left(\mathbb{I}_{m}+\omega K D(s)\right)^{-1}
$$

The $\mathcal{L}_{1}$ adaptive controller is defined by combining the adaptive control law (13), the predictor (11), the adaptation rule (12a). The design of $C(s)$ will be discussed in Section 3.6. 


\subsection{Discrete time implementation}

Since the controller shall be implemented in a computer hardware, the controller, i.e., the state predictor must be discretized.

Consider the continuous time state-predictor dynamics in (11)

$$
\begin{aligned}
\dot{\hat{x}}(t) & =A_{m} \hat{x}(t)+B_{m}\left(\omega_{0} u_{a d}(t)+\hat{\sigma}_{1}(t)\right)+B_{u m} \hat{\sigma}_{2}(t) \\
y(t) & =C_{m} \hat{x}(t)
\end{aligned}
$$

This can be written as, assuming zero-order hold for the input $u_{a d}(t)$ and $\hat{\sigma}_{i}(t)$,

$$
\begin{aligned}
\hat{x}(k+1) & =A_{m_{d}} \hat{x}(k)+B_{m_{d}}\left(\omega_{0} u_{a d}(k)+\hat{\sigma}_{1}(k)\right) \\
& +B_{u m_{d}} \hat{\sigma}_{2}(k) \\
y(k) & =C_{m_{d}} \hat{x}(k)
\end{aligned}
$$

where

$$
\begin{aligned}
A_{m_{d}} & =e^{A_{m} T_{s}} \\
B_{m_{d}} & =A_{m}^{-1}\left(e^{A_{m} T_{s}}-\mathbb{I}_{n}\right) B_{m} \\
B_{u m_{d}} & =\mathcal{N}\left(B_{m_{d}}^{T}\right) \\
\operatorname{rank}\left(\left[B_{m_{d}}, B_{u m_{d}}\right]\right) & =n \\
B_{d} & =\left[B_{m_{d}}, B_{u m_{d}}\right] \\
C_{m_{d}} & =C_{m}
\end{aligned}
$$

\section{ADAPTIVE BACKUP CONTROLLER DESIGN}

The $\mathcal{L}_{1}$ adaptive control with the modified piecewise constant adaptation law which was derived in the previous section has been used in this study to design robust adaptive backup flight control laws.

The controller has been implemented in Simulink, and then later code-generated to C-code in order to merge with the nonlinear high fidelity model. The following sections describe the process of tuning the controller to obtain the desired qualities.

\subsection{Flying qualities}

Before designing any flight control system the engineer needs to know what degree of control is required for the pilot to consider the aircraft safe and flyable. The flying qualities expected by the pilot depend mostly on the type of aircraft and the flight phase of the mission.

Flying qualities are largely related to the dynamic and control characteristics of the aircraft. If we for example consider the short-period motion as a second-order linear transfer function

$$
G(s)=\frac{\omega_{n}^{2}}{s^{2}+2 \zeta \omega_{n} s+\omega_{n}^{2}}
$$

The question that needs to be answered is what values should $\zeta$ (damping ratio) and $\omega_{n}$ (undamped natural frequency) have to satisfy the pilot. Researchers have studied this problem using simulators and flight test aircraft, and a common rating system is the Cooper-Harper scale [8]. Further guidelines from [8] and [4] were used in order to place the poles to achieve satisfactory flying qualities, see Section 3.3. They included requirements for dutch roll damping ratio and natural frequency and also the speed dependant time-constant of the roll response.

\subsection{Sampling frequency}

The piecewise adaptive law states that a higher sampling frequency results in better performance [7]. In this project a fixed sampling frequency of $60 \mathrm{~Hz}$ is chosen, which is a representative execution rate of the digital flight control laws on the JAS 39 Gripen fighter.

\subsection{Reference system design}

When designing the reference system for the statepredictor in (15) the method proposed by [3] is used to design a nominal feedback, $K_{x y}$, to get the desired response for the nominal linearised model. Compared to using a generic reference system to specify the response, this method takes the cross-coupling effects in the $B$ matrix into consideration.

The linearised short-period dynamics in the trimmed state of Mach $=0.6$ and Altitude $=5000 \mathrm{~m}$ were used as reference system and the nominal feedback were designed with damping $\zeta_{p_{H}}=0.9$, and $f_{n_{p_{H}}}=0.5 \mathrm{~Hz} \rightarrow \omega_{n_{p_{H}}}=$ $(0.5 \times 2 \pi) \mathrm{rad} / \mathrm{s}$.

The same methodology was adopted for the lateral dynamics.

\subsection{Model errors}

The uncertainties considered in this paper are

$$
\begin{aligned}
f_{\Delta}(t, x(t)) & =A_{\Delta}(t) x(t) \\
B_{\Delta}(t, x(t)) & =B_{\Delta}(t)
\end{aligned}
$$

which are variations of the system dynamics and control surface efficiency as the speed and altitude changes. The changes in the dynamics are assumed not to change with respect to the current states. Thus $f(t, x(t))$ from $(7)$ is given by

$$
\begin{aligned}
f(t, x(t))=\left(B_{i d}+\right. & \left.B_{\Delta}(t)\right) \sigma_{u}(t)+A_{\Delta}(t) x(t) \\
& +B_{\Delta}(t)\left(-K_{x y} x(t)+u_{a d}(t)\right)
\end{aligned}
$$

\subsection{Control surface dynamics}

Control surface dynamics is modelled with a first order low-pass filter with a bandwidth of $20 \mathrm{rad} / \mathrm{s}(\approx 3 \mathrm{~Hz})$. This is implemented in Simulink by adding a discrete time transfer function block to the input signal going to the state-predictor. The model from commanded surface deflection to actual deflection becomes

$$
\delta(z)=F(z) \delta_{c m d}(z)=\frac{0.2835}{z-0.7165} \delta_{c m d}(z)
$$

Rate limit and saturation blocks are also added to the control signal before the state-predictor, see Figure 2, to create an overall nonlinear model. Time-delays to the actuators have not been considered. The extension of considering actuator dynamics does not change the architecture [9], however the low-pass filters are now defined as

$$
C(z) \triangleq F(z) \omega K D(z)\left(\mathbb{I}_{m}+F(z) \omega K D(z)\right)^{-1}
$$


where $K$ and $D(z)$ need to ensure that $C(z)$ is a strictly proper and stable transfer function with static gain $C(0)=$ 1 for all $F \in \mathbb{F}_{\Delta}$.

\subsection{Low-pass filter bandwidth design}

For the proofs of stability and performance bounds, the choice of $K$ and $D(s)$ also needs to ensure that a certain $\mathcal{L}_{1}$-norm condition holds [5]. Specifying $K$ and $D(s)$ to get the desired filter $C(s)$ as in (20), while satisfying the $\mathcal{L}_{1}$-norm is an open problem.

We know from theory than the filters have to be BIBOstable with static gain $C(0)=1$ and not let frequencies higher that the effective control bandwidth through [5]. A large $K$ improves load disturbance attenuation but more measurement noise is injected, causing large actuator demands. This noise will be damped by the actuator and plant dynamics, but can cause actuator wear and undesired excitation of the plant dynamics [10]. One simple choice of $D(s)$ is $\frac{1}{s}$, which was chosen for this application.

It is important to notice that the stability and performance guarantees are only valid if the nonlinear functions $f_{i}(t, x)$ fit in to the assumptions that we have made. Reducing the $\mathcal{L}_{1}$-norms will make robustness to deviations that do not fit into (7) smaller [11]. The assumptions of the nonlinear functions do not consider for example time-delays in the system.

The low-pass filters designed for the $\mathcal{L}_{1}$ controller are first-order, discrete time filters with a sampling period of $1 / 60 \mathrm{~s}$. They are based on the $s$-domain filters, but discretized as below. A design was chosen to have separate filters for the matched and unmatched estimates. For the unmatched estimate a cascaded filter was used, inspired by [11] and [2]. This gives the following control law:

$$
\begin{aligned}
\hat{\sigma}_{2}(z) & =C_{u m_{1}}(z) \hat{\sigma}_{2}(z) \\
u(z) & =K_{g} r(z)-K_{x y} x(z)-C_{m}(z) \hat{\sigma}_{1}(z) \\
& -C_{u m_{2}}(z) H_{m}(z)^{-1} H_{u m}(z) \hat{\sigma}_{2}(z)
\end{aligned}
$$

\subsection{Multiple reference systems}

Simulations have shown that one controller can not cope with all the uncertainties throughout the entire flight envelope, especially in low speed flying (landing). An idea was developed to increase the number of controllers, one for low speed, low altitude (landing) and one for high speed, high altitude (normal flying). Switching between these two was done by knowing when the landing gear was down or up.

\section{RESULTS}

In Figure 3 step responses are presented for the baseline controller for the nominal model. The dotted lines can be seen as guidelines for the step responses, the values should be within the lines to be regarded to have adequate flying qualities, see Section 3.1. The figure illustrates that when the speed varies (the model changes), the step responses stabilize the system, but with poor or inadequate flying qualities.
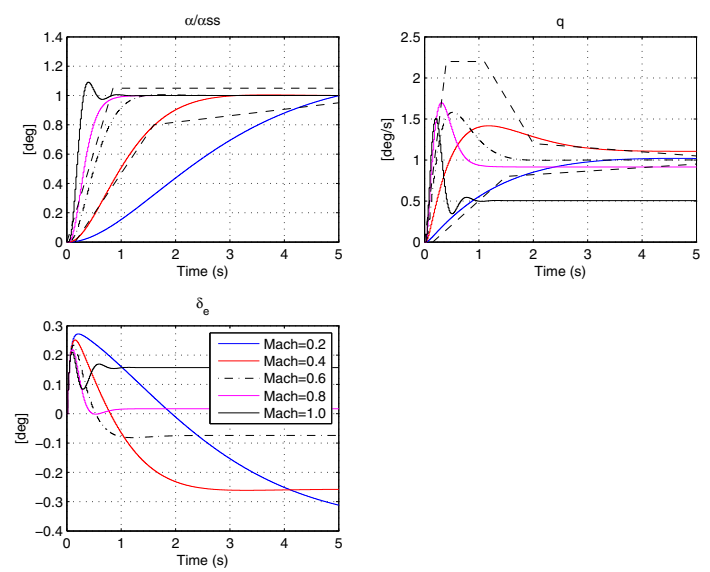

Fig. 3. Pitch responses for the baseline controller at different mach.

\subsection{VEGAS model simulations}

The high-speed and low-speed short-period and lateral controllers were tested with the nonlinear VEGAS model, the results of step responses of the high-speed controller can be found in Figure 4 and 5. Only the step responses with adequate flying qualities are shown. At $5000 \mathrm{~m}$, the high-speed controller manages to adapt to the uncertainties quite well down to $M a c h=0.3$.
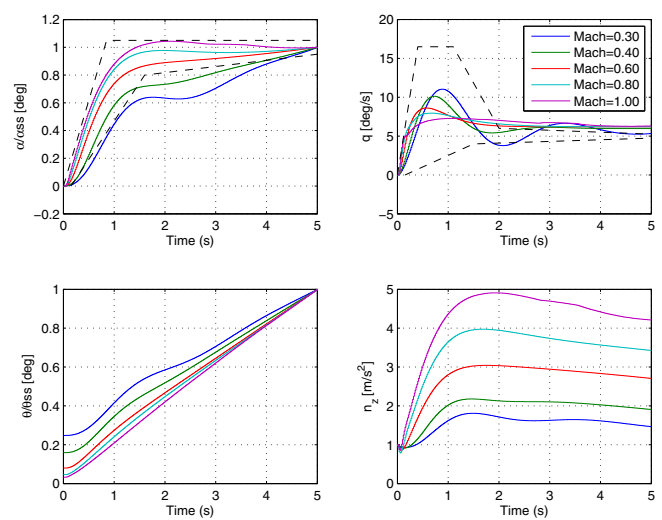

Fig. 4. Nonlinear step responses in pitch for augmented $\mathcal{L}_{1}$ controller designed for the high speed case.
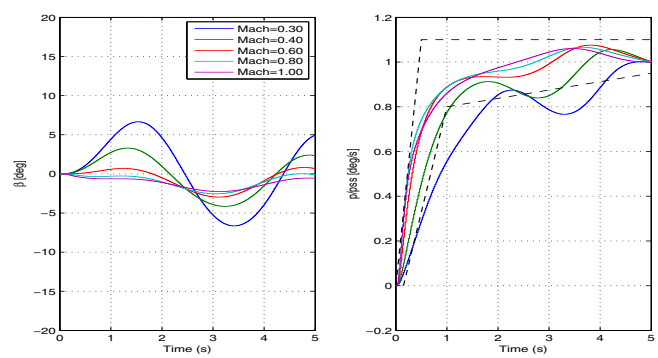

Fig. 5. Nonlinear step response in roll for augmented $\mathcal{L}_{1}$ controller designed for the high speed case.

Overall, the high-speed controller has a hard time to cope with the uncertainties related to the low speeds (Mach $\leq$ $0.3)$, the speeds very much critical for landing. 


\section{CONCLUSIONS}

The results are promising for this application, even with a limited adaptation rate of $60 \mathrm{~Hz}$, which might open new doors in exploring the benefits of adaptive control. One controller, with the modified piecewise constant adaptation law, with one reference system is not able to cope with all the uncertainties related to the loss of speed and altitude data. It increases robustness, but not for the whole flight envelope.

Because of the problems at low speeds, a switching strategy was formulated between two $\mathcal{L}_{1}$ adaptive controllers, without a theoretical foundation for this approach. It was however flown in a simulator with relative success. The high-speed controllers in both the longitudinal and lateral modes are effective down to around $M a c h=0.3$ if Altitude $\leq 5000 \mathrm{~m}$, and about Mach $=0.4$ at Altitude $>$ $5000 \mathrm{~m}$. The low-speed controllers, at least for the longitudinal case, can cope with speeds of about Mach $=0.15-$ 0.4 at Altitude $<3000 \mathrm{~m}$.

The tuning of the filters has been an open problem throughout the project, even if the control channel bandwidth limits the design. Trial and error methods have been adopted with inspiration from other peoples work to get a good balance between performance and robustness. The state-predictor was transformed to discrete time with a reformulation in the adaptive law. This structure has not been proven in theory, but has given satisfactory results in simulations.

Problems were most prominent with the lateral controller, the $\mathcal{L}_{1}$ adaptive controller had big problems in stabilizing the inner loops by itself. The addition of the baseline controller was very much needed to achieve acceptable performance. Overall, there were more issues related to the lateral MIMO controller than the longitudinal SISO one.

The baseline controller managed to provide similar performance in the design points of the flight envelope as the augmented controller, but in all other cases, the augmented controller improved the performance. Tuning was also done to reduce the bandwidth of the closed-loop desired system in the state-predictor to calm down the desired system response to be able to account for the lower speeds, without success. Overall the $\mathcal{L}_{1}$ adaptive controller with the modified piecewise constant adaptive law can be considered to increase robustness and performance inner loops in this application, with reservation for the lateral inner loops in low speeds. More work still needs to be done in order to integrate it into a complex flight control system.

\section{REFERENCES}

[ADM] Admire model. http://www.foi.se/admire, Accessed 2012-11-15.

[1] C. Cao and N. Hovakimyan. Design and analysis of a novel 11 adaptive controller, part i. In American Control Conferencel, pages 3397-3402, Minneapolis, Minnesota, USA, jun 2006.

[2] I. M. Gregory, E. Xargay, C. Cao, and N. Hovakimyan. L1 adaptive flight control system systematic design and verification and validation of control metrics. In $A I A A-2010$-77793, 2010.
[3] B. J. Griffin, J. Burken, and E. Xargay. L1 adaptive control augmentation system with application to the x-29 lateral/directional dynamics: A multi-input multi-output approach. In 2010 AIAA Guidance, Navigation, and Control Conference, 2010.

[4] K. S. Gunnarsson. Kravdokument för styrlagsdesign. Technical report, SAAB Aerosystems, 2001.

[5] N. Hovakimyan and C. Cao. L1 Adaptive Control Theory - Guaranteed Robustness with Fast Adaptation. Society for Industrial and Applied Mathematics, 1st edition, 2010. ISBN-13: 978-0-898717-04-4.

[6] N. Hovakimyan, C. Cao, E. Kharisov, E. Xargai, and I. M Gregory. L1 adaptive control for safety-critical systems. In IEEE Control Systems Magazine, 2010.

[7] Z. Li and N. Hovakimyan. L1 adaptive controller for mimo systems with unmatched uncertainties using modified piecewise constant adaptation law. In 51st IEEE Conference on Decision and Control (CDC), pages 7303-7308, 2012.

[8] R. C. Nelson. Flight Stability and Automatic Control. McGraw-Hill Book Co, 2 edition, 1998. ISBN-10: 007-115838-3.

[9] H. Øystein. L1 adaptive control of the inner control loops of an f-16 aircraft. Master's thesis, Norwegian University of Science and Technology, 2012.

[10] A. Pettersson, K.J. Åstrom, A. Robertsson, and R. Johansson. Analysis of linear 11 adaptive control architectures for aerospace applications. In IEEE 51st Annual Conference on Decision and Control (CDC), pages 1136-1141, 2012.

[11] A. Pettersson, K. J. Åström, A. Robertsson, and R. Johansson. Augmenting 11 adaptive control of piecewise constant type to a fighter aircraft. performance and robustness evaluation for rapid maneuvering. In 2012 AIAA Guidance, Navigation, and Control Conference, Minneapolis, Minnesota, 2012.

[12] J. L. Tyler. L1 adaptive control augmentation system for the x-48b aircraft. Master's thesis, University of Illinois at Urbana-Champaign, 2009.

[13] E. Xargay, N. Hovakimyan, and C. Cao. L1 adaptive controller for multi-input multi-output systems in the presence of nonlinear unmatched uncertainties. In 2010 American Control Conference, pages 874-879, 2010.

[14] E. Xargay, N. Hovakimyan, V. Dobrokhodov, R. B. Statnikov, I. Kaminer, C. Cao, and I. M. Gregory. Flight test of 11 adaptive controller on the nasa airstar flight test vehicle. In AIAA Guidance, Navigation and Control Conf., AIAA-2010-8015, 2010. 